\title{
A Study on Threats and Possible Approaches for Microgrid Safety and Control
}

\author{
Avinash R \\ Assistant Professor \\ Department of EEE \\ Vidyavardhaka College of Engineering \\ Mysuru, Karnataka, India
}

\author{
Dr. Shobha Shankar \\ Professor \& Head \\ Department of EEE \\ Vidyavardhaka College of Engineering \\ Mysuru, Karnataka, India
}

\author{
Goutham B \\ Assistant Professor \\ Department of EEE \\ Vidyavardhaka College of Engineering \\ Mysuru, Karnataka, India
}

\begin{abstract}
Microgrids with a potential structure developed as a system that combines renewable energy sources, energy storage systems and loads. Microgrids are capable of improving the quality, Reliability and efficiency of the power delivered to the consumers both in grid connected and Islanded mode. However, when implementing microgrids, a variety of problems concerning the security issues have to be handled. The existing modes of operation and the existing DERs are capable of causing the traditional security schemes to be unproductive. Therefore, novel security approaches have been developed. Thus, in this paper a complete review on the modern security approaches is discussed considering the security issues explained above. Prominence to surveys related to adaptive protection is exhibited in this paper, which are believed as favourable solution for microgrid safety and control. The paper briefly presents a microgrid structure and addresses the safety and control challenges observed in the existing Microgrid. Later the paper focuses on overview of Adaptive protection and the possible novel protection schemes for microgrid safety and control found in the technical research articles. However, it can be noticed that efforts are still required to attain a complete solution for the safety and control challenges observed in the existing Microgrid.
\end{abstract}

Keywords: Microgrids, Meta-heuristics, Computational Intelligence, Neural Network Topology-Adjusted Algorithm, DGs, Microgrid Protection schemes

\section{INTRODUCTION}

As we know that Economic development of a country is greatly influenced by the access to electricity by the people of that country. Efforts have been made in recent years to provide people with unfailing supplies of electricity. Even then there are regions which are still under-served. Many development agencies and governments intervene to improve electricity access to such regions of the country, this intervention would have a casual effect on penury, the economic growth and other development indicators of the country. As per the "The Energy Progress Report-2020" by SDG-7, 789 million people across the world do not have access to electricity as on 2018. Microgrids nowadays find great demand as a solution for the above said problem. The microgrid concept dates back to 1882 , They integrate with
Distributed Energy Resources (DERs) and supply power in emergencies and in island connected mode. Integration of Microgrids with DERs introduce a number of challenges in the design of control and protection systems to provide reliable power to the consumers. In this paper we will look into challenges that arise in the protection system of Microgrids and possible solutions.

A microgrid can effectively integrate diverse sources of distributed generation (DG), They are capable of operating either connected to the grid or isolated from the grid. Microgrids have the facility to control the distributed generation locally, thereby decreasing the requirement of Central dispatch. Two major advantages of Microgrids are:

1. They can operate efficiently and safely acting as a single producer with its local distribution network.

2. They provide uninterruptible power supply with agreed power quality level to the loads with high priority / sensitivity. Thus, increasing reliability of the system.

Microgrids, and the introduction of DER units in general, pose a range of operational difficulties which have to be addressed in developing control and security systems to ensure that the necessary performance standards are not significantly impacted and the potential benefits of Distributed Generation (DG) units are fully exploited.

Some of these challenges emerge from inferences traditionally applied to modern distribution systems that are no longer true, whereas others originate from performance issues previously found only at the level of the transmission system.

At the present microgrid safety and control challenges include:

\section{A. Variations in fault currents}

The amplitude of Fault current in a Microgrid is dependent on the prevailing causes of short circuit. Apparently once the MG is operating in grid-connected condition, the level of short circuit will be higher in the transmission / distribution network when compared to 
the level of fault current when the microgrid is operating in quarantined condition.

\section{B. Delayed/Blinding of protection}

Introducing $\mathrm{DG}$ to the microgrids results in increased fault current levels of the network. This change in fault current levels modifies the ability of the protective device to discriminate which results in sympathetic tripping of healthy part in the network when the occurrence of fault is in another section of the network. This causes uncalled loss of power supply to the consumers of a healthy line; this kind of unwanted protection is referred to as blinding protection.

\section{Uncoordinated and Automatic reclosing}

The introduction of DG into MG causes mal function of the protective devices created by auto reclosures due to improper coordination between the relays and Circuit breakers and other protective devices.

\section{Nuisance tripping}

A Nuisance tripping arises when a protective device associated to one branch of the network reacts to a fault in a neighbouring branch of the same network, when a DG is inserted to the existing Micro grid network.

\section{E. Issues related to communication and Cyber attacks}

A microgrid needs proper communication system in order to ensure proper coordination between the grid and the protective devices such as relays and Circuit breakers. Microgrid uses communication system to remotely control the relays and isolators. These systems should provide fast and reliable communication between the Microgrid and the protective devices. If these systems are not provided with proper security, the hacker can hack the system and Send messages control the protective devices or can inject a virus into the system which can help the hacker study the system or to breakdown the system in no time.

If we look at the history a Russian Hacker group was successful in hacking the Ukrainian power grid and were able to actuate all the relays and opened the breakers in the substation, which affected more than two lakh customers.

\section{F. Issues related to Energy Storage Systems}

ESS in microgrids find their application in storing energy during off peak hours and supplying the stored power during peak hours. The current ESS technologies fail to meet the requirements of the microgrids due to various issues such as Size and Capacity, Cost, reliability of the system, charging and discharging rate and Energy Management [10].

As explained above the existing operating approaches and the incidence of DERs may make traditional protection schemes unproductive. The above challenges demand approaches with modern protection schemes. The paper presents different modern-day approaches which are based on sophisticated algorithms, communications and shared information. Also, multiple generating units are connected in a microgrid which enables multidirectional flow of power because of which the fault cannot be assumed to originate from a single source. Thus, the generating units should be enabled with fault ride through facilities which has to be done for both the operating modes.

\section{APPROACHES BASED ON FIELD PROGRAMABLE GATE ARRAY (FPGA) [1]}

In the existing distribution network, we make use of over current relays. We know that the direction of current flow changes with change in modes of operation of a microgrid, therefore it is a must to incorporate directional over current relays in the system. The directional feature in the existing over current relays can be implemented using FPGA programming, this is called as soft core directional over current relay. The proposed FPGA-based DOCR comprises of several functional blocks such as ADC, DFT filter and RMS estimation, ZCD, PD, REM, and power system module (PSM). In this method a digital module was developed to detect the phase, also a parallel design was done to compute the tripping time using the Integer arithmetic Verilog hardware description language platform on the Virtex ML505 FPGA development board. Now the performance of the DOCR is tested for faults created in different locations with different time dial settings. Then the HIL (Hardware in loop) verification was of the new DOCR is done for different fault conditions.

\section{APPROACHES BASED ON COMPUTATIONAL INTELLIGENCE METHODS.}

Distributed generators are the key components of a microgrid. The existing DGs have unidirectional current flow and hence over current protection schemes are employed in the existing microgrid systems. With the integration of DG, the power flow in the network changes makes the existing protection scheme unsuitable. It is mandatory that the microgrid which operates as a single entity when operating with the main grid should have a protection scheme which is capable of:

1. Isolating itself from the main grid in case of fault occurrence in the main grid.

2. Localising the fault created in the Microgrid within the network.

To do this the protection scheme should be carefully designed to ensure proper Sensitivity and Selectivity, which helps in improving the reliability of the microgrid.

Tang and Yang [2] in their article explain about the strategies involved in the over current protection for distributed systems with Distributed generators using fault current limiters. In this method each relay is considered as an Intelligent Electrical Device incorporated with the ability to communicate with their peers and identify their state of operation with reference to the decision tree which sets the operation and the Neural Network Topology-Adjusted Algorithm (NNTA) [3] model based on the data processed 
by the Fast Fourier Transform analysis. The advantage with this approach is that the time consumed for practical application is curtailed, this makes the application of this method easy for the system with Fault Current Limiters and Distributed Generators.

\section{APPROACH BASED ON STATE-OF-THE-ART ARTIFICIAL INTELLIGENCE METHODS [6]}

Nowadays the energy sector is rapidly changing and the topology of the network is getting complex day by day, to cope up with the rapidly changing energy sector, intelligent systems are essential to manage the system and to take timely decisions. Even though there are many AI techniques such as ANN, Genetic Algorithm, Reinforcement Learning and Multi agent systems which can be used for load forecasting, Networking, Optimisation and design control strategies. But many of the system operations are performed manually, this can be overcome using AI techniques. The future energy system will be complex and there is a need for advanced automatic controllable resources, these techniques would be able to reduce the cost and make the system to develop efficient and incorporate powerful means to handle the constraints of the conventional microgrids

\section{APPROACHES BASED ON META-HEURISTIC AND EVOLUTIONARY ALGORITHMS [7]}

The majority of industrialized and developing economies now have renewable energy generating stations built and run successfully. Integration of DG to the existing Microgrids reduces the carbon footprint globally, but faces several challenges, one such challenge is the issues with protection coordination. When compared to the fault current in the system when the microgrid is operating in the islanded mode varies with that fault current which occurs when the Microgrid has DG integrated to it. In this case the operating time of the over current relays changes which causes miscoordination, leading to the mal operation of the protective devices and may cause Blinding protection at the later stage.

Recalibrating the settings of each over current relay for each network emergency viz., line outage, coupling or decoupling of DG, swap between microgrid's operation between grid connected mode to islanded mode. Thus, a model that takes into account all operating emergencies which are suitable for $\mathrm{N}-1$ contingencies plays a key role in over current relay coordination.

In the Metaheuristic approach, A single set of overcurrent relay settings, which are good enough to keep the over current relays operational for all kinds of network topologies is explained using invasive weed optimization (IWO) [8] - linear interval programming (ILP) technique. In this hybrid optimiser method, the miscoordination problem is modelled using ILP and solved using IWO technique. The results of this technique are promising and provides results faster with fewer number of generations.

\section{APPROACHES BASED ON MULTI-AGENT SYSTEMS [9]}

A typical LVMG (Low Voltage Micro grid) can be controlled using a Microgrid central controller placed at the LV side of the Medium voltage or Low voltage substations. Two other controllers namely Load controller and the Micro source controllers are used to control the loads connected and the Micro sources, respectively. These controllers are also facilitated with narrow band communication link to enable them to exchange the necessary information with the main controller i.e. Microgrid central controller. In the decentralised multi agent approach the restoration action sequence of the microgrid after the fault, is determined by the online data related to the power generated and the load consumption. To do this the microgrid components such as micro sources, storage devices, and loads are dynamically modelled and then using the distributed averaging method, decentralized multi-agent-based approach to restore the Microgrid loads and generations with a proper sequence of actions after a total blackout is designed.

\section{CONCLUSION}

This paper described the challenges that are faced by present Micro-grids when integrated with DGs, also the paper explained about all the existing and possible solutions, their methodologies to improve the security and reliability of the system. Even though we have number solutions for the protection problems, there are restraints in the available methods in terms of accuracy and time of response. In order to overcome these limitations, there is a need to develop Hybrid methods or adaptive methods so that the protective system will be able to provide suitable protection by adapting to different kinds of faults and updating the system with new protection schemes. Artificial intelligence plays the key role in designing the adaptive systems, Artificial intelligence systems can be incorporated in the protective system design, so that the system can learn different faults and can analyse the causes of the fault as and when they occur. At the later stage the system can update the system for the new fault case and can use this reference in future when a similar kind of fault occurs. Thus, Incorporating Artificial intelligence in addition to the available Heuristic algorithms in the existing protective system can improve the stability and reliability of the system.

\section{REFERENCES}

[1] Praveen Kumar, Vishal Kumar \& Rajendra Pratap (2019) FPGA Based Design and HIL Verification of a Soft-Core Directional-OCR, Electric Power Components and Systems, 47:14-15, 1423-1436, DOI: 10.1080/15325008.2019.1661548

[2] Tang W-J, Yang H-T. Self-adaptive protection strategies for distribution system with DGs and FCLs based on data mining and neural network. In: 2017 IEEE international conference on environment and electrical engineering and 2017 IEEE industrial and commercial power systems Europe (EEEIC/I\&CPS Europe). IEEE; 2017. https://doi.org/10.1109/eeeic.2017.7977738

[3] Wen-Jun Tang and Hong-Tzer Yang "Data Mining and Neural Networks Based Self Adaptive Protection Strategies for Distribution Systems with DGs and FCLs"2018 Special Issue $17^{\text {th }}$ International Conference on Environment and Electrical Engineering. https://doi.org/10.3390/en11020426 
[4] Thararak, Panida \& Jirapong, Peerapol. (2020). Implementation of Optimal Protection Coordination for Microgrids with Distributed Generations Using Quaternary Protection Scheme. Journal of Electrical and Computer Engineering,2020,1-13. https://doi.10.1155/2020/2568652.

[5] R. Jain, D. L. Lubkeman and S. M. Lukic, "Dynamic Adaptive Protection for Distribution Systems in Grid-Connected and Islanded Modes," in IEEE Transactions on Power Delivery, vol. 34, no. 1, pp. 281-289, Feb. 2019, doi: 10.1109/TPWRD.2018.2884705.

[6] MDPI and ACS Style, Ali, S.S.; Choi, B.J. "State-of-the-Art Artificial Intelligence Techniques for Distributed Smart Grids: A Review," Electronics 2020, 9, 1030.

[7] S. T. P. Srinivas and K. S. Swarup, "Optimal Protection Coordination for Microgrid Considering DG and Line Outages Using IWO-ILP Technique," 2018 20th National Power Systems Conference (NPSC), Tiruchirappalli, India, 2018, pp. 1-6, doi: 10.1109/NPSC.2018.8771734.

[8] Kumar D., Gandhi B.G.R., Bhattacharjya R.K. (2020) Introduction to Invasive Weed Optimization Method. In: Bennis F., Bhattacharjya $R$. (eds) Nature-Inspired Methods for Metaheuristics Optimization. Modelling and Optimization in Science and Technologies, vol 16. Springer, Cham. https://doi.org/10.1007/978-3-030-26458-1_12

[9] Rokrok, Ebrahim, et al. "A decentralized multi-agent-based approach for low voltage microgrid restoration." Energies 10.10 (2017): 1491.

[10] M. Faisal, M. A. Hannan, P. J. Ker, A. Hussain, M. B. Mansor and F. Blaabjerg, "Review of Energy Storage System Technologies in Microgrid Applications: Issues and Challenges," in IEEE Access, vol. $6, \quad$ pp. 35143-35164, 2018, doi: 10.1109/ACCESS.2018.2841407.

\section{AUTHORS PROFILE}

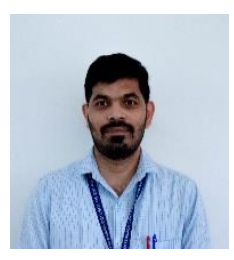

Prof. Avinash R, completed M. Tech in Power Electronics from Reva Institute of technology and Management, Bengaluru, India. He is currently working as an Assistant Professor in the department of Electrical and Electronics Engineering at Vidyavardhaka College of Engineering Mysuru, Karnataka. Areas of interest include Microgrids, Power quality and Power system optimisation techniques

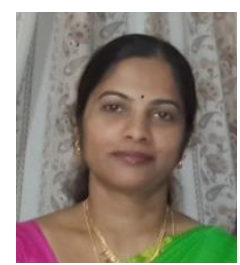

Dr. Shobha Shankar, is currently working as Professor and HOD in the Department of Electrical and Electronics Engineering at Vidyavardhaka College of Engineering Mysuru, Karnataka. Areas of interest include AI applications to Power System, Power Quality, Renewable Energy integration to grid.

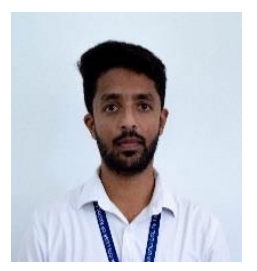

Prof. Goutham B, completed M. Tech in Computer Application in Industrial Drives from Shri Siddartha Academy of Higher Education, Tumkur, India in 2015. He is currently working as an Assistant Professor in the Department of Electrical and Electronics Engineering at Vidyavardhaka College of Engineering Mysuru, Karnataka. Areas of interest include Smart Grids, Cyber Security, Microgrids, Renewable Energy sources, Electrical Machines. 